Research Article

\title{
Two Extensions of the Quadratic Nonuniform B-Spline Curve with Local Shape Parameter Series
}

\author{
Xiang Kong and Jun Chen \\ Faculty of Science, Ningbo University of Technology, Ningbo 315211, China \\ Correspondence should be addressed to Jun Chen; chenjun88455579@163.com
}

Received 25 March 2021; Revised 24 June 2021; Accepted 21 July 2021; Published 3 September 2021

Academic Editor: Nickolas S. Sapidis

Copyright (c) 2021 Xiang Kong and Jun Chen. This is an open access article distributed under the Creative Commons Attribution License, which permits unrestricted use, distribution, and reproduction in any medium, provided the original work is properly cited.

\begin{abstract}
Two extensions of the quadratic nonuniform $B$-spline curve with local shape parameter series, called the $W^{3} D^{3} C^{1} P^{2}$ spline curve and the $W^{3} D^{4} C^{2} P^{1}$ spline curve, are introduced in the paper. The new extensions not only inherit most excellent properties of the quadratic nonuniform $B$-spline curve but also can move locally toward or against the fixed control polygon by varying the shape parameter series. They are $C^{1}$ and $C^{2}$ continuous separately. Furthermore, the $W^{3} D^{3} C^{1} P^{2}$ spline curve includes the quadratic nonuniform $B$-spline curve as a special case. Two applications, the interpolation of the position and the corresponding tangent direction and the interpolation of a line segment, are discussed without solving a system of linear functions. Several numerical examples indicated that the new extensions are valid and can easily be applied.
\end{abstract}

\section{Introduction}

The nonuniform polynomial $B$-spline curve has been popularly applied in computer-aided geometric design (CAGD) [1]. In particular, the quadratic one, which is the simplest nonuniform $B$-spline curve, has gained widespread application based on its efficiency and convenience.

However, with the specified parameterization method of control point, the position of the classical $B$-spline curve is determined by the control polygon. The only way to adjust the shape of the curve is the modification of the control points, which can be seen as a shortcoming for shape adjusting in some cases. For example, in the shape designing, the control polygon as the outline curve is given firstly. Then, the unique $B$-spline curve, which is smooth and approaches to the control polygon, can be obtained correspondingly. In fact, when the designer specifies the control polygon, he or she may already be satisfied with the rough shape of curves they wanted. Hence, the designer prefers to get more candidate curves characterized by the given control polygon which keeps the original rough shape. In other words, the curve should be modified tinily with the same control polygon.
To overcome the rigidity of the nonuniform polynomial $B$-spline curve, the nonuniform rational $B$-spline (NURBS) curve is developed by assigning a weight for each control point. Therefore, the NURBS curve can be modified by altering the weights with fixed control polygon. However, in the light of the complexity of fractional expressions, it also suffers from several new drawbacks, such as difficulty in choosing the value of the weights, unstability with the increasing order of rational fraction, and trouble in derivatives and integrals computation. Furthermore, changing the weights to adjust the shape of a NURBS curve is hard to the designer [2].

In order to avoid the inconveniences of the NURBS curve, some new spline curves, the expressions of which include the shape parameters, have been extensively studied. Thus, these new spline curves can be altered by the shape parameters effectively without changing the control polygon. In [3], the cubic $\beta$-spline curve with two global shape parameters was presented, which is $C^{2}$ continuous for a uniform knot sequence. The CB-spline curve or HB-spline curve constructed in the space spanned by polynomial and trigonometric/hyperbolic functions is another feasible method [4-8]. The definition domain can be seen as a global 
shape parameter. In [9], the variable degree polynomial spline curve with two degrees which play the role of design parameters was described. In [10], using a simple modification of the well-known geometric construction of $C^{4}$ quintic splines, a class of $C^{2} \cap F C^{3}$ spline curves possessing tension properties is described. In $[11,12]$, the shape parameters were given by blending the $B$-spline curves and a singularly parameterized sequence of connected line segments. In [13-15], with the help of the blending functions, the shape parameter series were incorporated into the usual spline basis functions, and consequently several kinds of the piecewise quartic polynomial spline curves with shape parameters were investigated under the uniform/nonuniform knot sequence.

The main purpose of this paper is to increase the flexibility of the widely used quadratic nonuniform $B$-spline curve by imitating the way of [13-15]. Two kinds of nonuniform piecewise polynomial spline curve with local shape parameter series, called the $W^{3} D^{3} C^{1} P^{2}$ spline curve and the $W^{3} D^{4} C^{2} P^{1}$ spline curve, are introduced in the present paper. They are considered as the extensions of the quadratic nonuniform $B$-spline curve, because of the following:

(i) They have three consecutive control points for each curve segment, which means that they have the same structure of the expression as the quadratic $B$ spline curve

(ii) They inherit the most excellent geometric properties of the quadratic $B$-spline curve, such as the properties of geometric invariance, affine invariance, local support, convex hull, variation diminishing (VD), as well as $C^{1} / C^{2}$ continuity

(iii) They include the quadratic nonuniform $B$-spline curve as a special case for the $W^{3} D^{3} C^{1} P^{2}$ spline curve

(iv) They can be adjusted locally by varying the values of shape parameters with the fixed control polygon, which exceed the quadratic nonuniform $B$-spline curve

When the shape parameter is global, the change of a shape parameter value affects the entire curve leading to a global change, which is not so suitable for CAGD applications. In view of this, the shape parameters in the present paper are local. Varying a shape parameter value only allows modifying the shape of the curve locally in two neighboring curve segment. In addition, these two extensions can be closer to or more far away from the given control polygon than the quadratic $B$-spline curves with appropriate shape parameters.
The rest of this paper is organized as follows. In Section 2, two kinds of basis functions with local shape parameter series are described and the properties of them are analyzed in detail. The corresponding spline curves are constructed, and the local control of them is shown in Section 3. Open and closed spline curves are illustrated. Section 4 deals with two interpolations without solving a system of linear functions, the interpolation of the position and the corresponding tangent direction, and the interpolation of a line segment. The problems with the clamped knot vector are discussed in Section 5. Conclusion is given in Section 6. Our results are supported by numerical examples in Section 2, Section 3, Section 4, and Section 5.

\section{Basis Functions}

2.1. Definition. Given a nonuniform knot sequence $\left\{u_{i}\right\}_{i=-\infty}^{+\infty}$, let $\cdots<u_{0}<u_{1}<\cdots<u_{n-1}<u_{n}<\cdots$, where we refer to $\mathbf{U}=$ $\left(\ldots, u_{0}, u_{1}, \ldots, u_{n-1}, u_{n}, \ldots\right)$ as a knot vector. The piecewise linear transformation function $t(u)$ can transform the point in the interval $\left[u_{i}, u_{i+1}\right)$ to the one in the unit interval $[0,1)$ as follows:

$$
t(u)=\left\{\begin{array}{l}
\vdots \\
t_{i}(u), \quad u \in\left[u_{i}, u_{i+1}\right), \\
t_{i+1}(u), \quad u \in\left[u_{i+1}, u_{i+2}\right), \quad t_{i}(u)=\frac{u-u_{i}}{u_{i+1}-u_{i}} \\
t_{i+2}(u), \quad u \in\left[u_{i+2}, u_{i+3}\right) \\
\vdots
\end{array}\right.
$$

With the help of the linear transformation function $t(u)$, two kinds of piecewise polynomial basis functions with local shape parameter series are defined as follows.

Definition 1. Given a knot vector $\mathrm{U}$, the $W^{3} D^{3} C^{1} P^{2}$ basis functions $\left\{N_{I, i}(u)\right\}_{i=-\infty}^{+\infty}$ with two local shape parameter series $\left\{\alpha_{i}\right\}_{i=-\infty}^{+\infty}$ and $\left\{\beta_{i}\right\}_{i=-\infty}^{+\infty}$ and the $W^{3} D^{4} C^{2} P^{1}$ basis functions $\left\{N_{I I, i}(u)\right\}_{i=-\infty}^{+\infty}$ with one local shape parameter series $\left\{\gamma_{i}\right\}_{i=-\infty}^{+\infty}$ are defined to be the following functions:

$$
N_{e, i}(u)=\left\{\begin{array}{ll}
N_{0}^{e, i}(t(u)), & u \in\left[u_{i}, u_{i+1}\right), \\
N_{1}^{e, i}(t(u)), & u \in\left[u_{i+1}, u_{i+2}\right), \\
N_{2}^{e, i}(t(u)), & u \in\left[u_{i+2}, u_{i+3}\right), \\
0, & u \notin\left[u_{i}, u_{i+3}\right),
\end{array} \quad(e=\mathrm{I}, \mathrm{II}),\right.
$$

where 


$$
\begin{aligned}
& \left(\begin{array}{c}
N_{0}^{I, i}(t) \\
N_{1}^{I, i}(t) \\
N_{2}^{I, i}(t)
\end{array}\right)=\left(\begin{array}{cccc}
0 & 0 & \alpha_{i}\left(1+d_{i}\right)-\beta_{i} d_{i} & \alpha_{i} \\
\alpha_{i} & \beta_{i} & 1-\alpha_{i+1}\left(1+d_{i+1}\right)+\beta_{i+1} d_{i+1} & 1-\alpha_{i+1} \\
1-\alpha_{i+1} & 1-\beta_{i+1} & 0 & 0
\end{array}\right)\left(\begin{array}{c}
B_{0}^{3}(t) \\
B_{1}^{3}(t) \\
B_{2}^{3}(t) \\
B_{3}^{3}(t)
\end{array}\right), \\
& 0<\alpha_{i}<1, \quad \max \left\{0, \frac{\alpha_{i}\left(1+d_{i}\right)-1}{d_{i}}\right\}<\beta_{i}<\min \left\{\frac{\alpha_{i}\left(1+d_{i}\right)}{d_{i}}, 1\right\}, \\
& \left(\begin{array}{c}
N_{0}^{I I, i}(t) \\
N_{1}^{I I, i}(t) \\
N_{2}^{I I, i}(t)
\end{array}\right)=\left(\begin{array}{ccccc}
0 & 0 & 0 & m_{14}^{i} & \gamma_{i} \\
\gamma_{i} & m_{22}^{i} & 1 & m_{24}^{i} & 1-\gamma_{i+1} \\
1-\gamma_{i+1} & m_{32}^{i} & 0 & 0 & 0
\end{array}\right)\left(\begin{array}{c}
B_{0}^{4}(t) \\
B_{1}^{4}(t) \\
B_{2}^{4}(t) \\
B_{3}^{4}(t) \\
B_{4}^{4}(t)
\end{array}\right) \\
& m_{14}^{i}=\frac{1}{2} \frac{\gamma_{i}+2 d_{i} \gamma_{i}+d_{i}^{2} \gamma_{i}-d_{i}^{2}}{1+d_{i}} \\
& m_{24}^{i}=-\frac{1}{2} \frac{-2+\gamma_{i+1}+2 d_{i+1} \gamma_{i+1}-d_{i+1}^{2}+d_{i+1}^{2} \gamma_{i+1}-2 d_{i+1}}{1+d_{i+1}}, \\
& m_{22}^{i}=\frac{1}{2} \frac{\gamma_{i}+2 d_{i} \gamma_{i}+d_{i}^{2} \gamma_{i}+d_{i}^{2}}{d_{i}\left(1+d_{i}\right)} \\
& m_{32}^{i}=-\frac{1}{2} \frac{\gamma_{i+1}-2 d_{i+1}+2 d_{i+1} \gamma_{i+1}-d_{i+1}^{2}+d_{i+1}^{2} \gamma_{i+1}}{d_{i+1}\left(1+d_{i+1}\right)}, \\
& \frac{d_{i}^{2}}{\left(1+d_{i}\right)^{2}}<\gamma_{i}<1-\frac{1}{\left(1+d_{i}\right)^{2}} \text {, } \\
& d_{i}=\frac{u_{i+1}-u_{i}}{u_{i+2}-u_{i+1}}, \\
& B_{i}^{n}(t)=\left(\begin{array}{c}
n \\
i
\end{array}\right)(1-t)^{n-i} t^{i}, \quad i=0,1, \ldots, n .
\end{aligned}
$$

The implication of the acronym $\mathrm{W}^{i} \mathrm{D}^{j} \mathrm{C}^{k} \mathrm{P}^{l}$ is shown in Table 1. More details of the properties mentioned in Table 1 can be seen in Section 2.2.

2.2. Properties. If the control polygon is maintained, the properties of the spline curve depend on its basis functions. To analyze the spline curve in Section 3, we list the properties of the $W^{3} D^{3} C^{1} P^{2}$ and the $W^{3} D^{4} C^{2} P^{1}$ basis functions $\left\{N_{e, i}(u)\right\}_{i=-\infty}^{+\infty}(e=\mathrm{I}, \mathrm{II})$ as follows, all of which can be proved from a direct calculation.

Property 1 (local support). For $u \in\left[u_{i}, u_{i+1}\right)$, the basis functions $\left\{N_{e, i}(u)\right\}_{i=-\infty}^{+\infty}(e=\mathrm{I}, \mathrm{II})$ are zero, which means that the width of the support intervals is three.

Property 2 (nonnegativity). For $u \in\left[u_{i}, u_{i+3}\right)$, the basis functions $N_{e, i}(u) \geq 0$ (e= I, II) if inequalities (4) or (6) hold.
Property 3 (normalization). The basis functions $\left\{N_{e, i}(u)\right\}_{i=-\infty}^{+\infty}(e=\mathrm{I}, \mathrm{II})$ satisfy

$$
\sum_{i=-\infty}^{+\infty} N_{e, i}(u)=1, \quad(e=\mathrm{I}, \mathrm{II}) .
$$

Property 4 (linear independence). The $W^{3} D^{3} C^{1} P^{2}$ basis functions $\left\{N_{I, i}(u)\right\}_{i=-\infty}^{+\infty}$ are linear independent if inequalities (4) and (8) hold; the $W^{3} D^{4} C^{2} P^{1}$ basis functions $\left\{N_{I I, i}(u)\right\}_{i=-\infty}^{+\infty}$ are linear independent if inequality (6) holds.

$$
\frac{\alpha_{i-1}}{1-\alpha_{i-1}} \neq \frac{\beta_{i-1}}{1-\beta_{i-1}} \text { or } \frac{\alpha_{i}}{1-\alpha_{i}} \neq \frac{\alpha_{i}\left(1+d_{i}\right)-\beta_{i} d_{i}}{1-\alpha_{i}\left(1+d_{i}\right)+\beta_{i} d_{i}} \text {. }
$$

Property 5 (continuity). The $W^{3} D^{3} C^{1} P^{2}$ basis functions $\left\{N_{I, i}(u)\right\}_{i=-\infty}^{+\infty}$ are $C^{1}$ continuous, and the $W^{3} D^{4} C^{2} P^{1}$ basis functions $\left\{N_{\mathrm{II}, i}(u)\right\}_{i=-\infty}^{+\infty}$ are $C^{2}$ continuous. 
TABLe 1: The implication of the acronyms $\mathrm{W}^{i} \mathrm{D}^{j} \mathrm{C}^{k} \mathrm{P}^{l}$.

\begin{tabular}{lcc}
\hline Acronyms & $W^{3} D^{3} C^{1} P^{2}$ basis functions & $W^{3} D^{4} C^{2} P^{1}$ basis functions \\
\hline$W:$ width of the support intervals & 3 & 3 \\
$D:$ degree of polynomial basis functions & 3 & 4 \\
$C:$ continuity of the basis functions & $C^{1}$ & $C^{2}$ \\
$P:$ number of local shape parameter series & 2 series: $\left\{\alpha_{i}\right\},\left\{\beta_{i}\right\}$ & 1 series: $\left\{\gamma_{i}\right\}$ \\
\hline
\end{tabular}

Property 6 (degeneracy). If $\alpha_{i}=d_{i} / 1+d_{i}, \beta_{i}=d_{i}+(2 / 3) /(1$ $\left.+d_{i}\right)$, the $W^{3} D^{3} C^{1} P^{2}$ basis functions $\left\{N_{\mathrm{I}, i}(u)\right\}_{i=-\infty}^{+\infty}$ degenerate into the quadratic nonuniform $B$-spline basis functions.

We illustrate the basis functions $\left\{N_{e, i}(u)\right\}_{i=-\infty}^{+\infty}(e=\mathrm{I}, \mathrm{II})$ in Figure 1.

2.3. The Case of Multiple Knots. So far, in Definition (1), we have assumed that each point is simple. We discuss the multiple knots with multiplicity $k(k=2,3)$ in this section. The construction of basis functions with multiple knots is analogous to Definition (2). The only difference between them is that we delete the corresponding pieces if the knot is multiple. For example, when $u_{i_{0}}=u_{i_{0}+1}$ is a double knot (here, we set $d_{i_{0}-1}=$ 0 to make sense of the corresponding definition), we define the basis functions $N_{e, i_{0}-1}(u)(e=\mathrm{I}, \mathrm{II})$ as follows:

$$
N_{e, i_{0}-1}(u)= \begin{cases}N_{0}^{e, i_{0}-1}(t(u)), & u \in\left[u_{i_{0}-1}, u_{i_{0}}\right), \\ N_{2}^{e, i_{0}-1}(t(u)), & u \in\left[u_{i_{0}+1}, u_{i_{0}+2}\right), \quad(e=\mathrm{I}, \mathrm{II}), \\ 0, \quad u \notin\left[u_{i_{0}-1}, u_{i_{0}+2}\right), & \end{cases}
$$

where we delete the piece on $\left[u_{i_{0}}, u_{i_{0}+1}\right)$.

According to the definition above, the support intervals width of the basis functions is reduced from 3 segments to $3-k$.

Property 7 (continuity at double knots). Suppose that $u_{i_{0}}=$ $u_{i_{0}+1}$ in the knot sequence, the basis functions $\left\{N_{e, j}(u)\right\}_{j=i_{0}-2}^{i_{0}}(e=\mathrm{I}, \mathrm{II})$ are $\mathrm{C}^{0}$ continuous at $u=u_{i_{0}}$ when $\alpha_{i_{0}-1}=1, \alpha_{i_{0}}=0(e=I)$ or $\gamma_{i_{0}-1}=1, \gamma_{i_{0}}=0(e=\mathrm{II})$.

Property 8 (discontinuity at triple knots). Suppose that $u_{i_{0}}=$ $u_{i_{0}+1}=u_{i_{0}+2+1}$ in the knot sequence, the basis functions $\left\{N_{e, j}(u)\right\}_{j=i_{0}-2}^{i_{0}^{+}+1}(e=\mathrm{I}, \mathrm{II})$ are discontinuous at $u=u_{i_{0}}$.

Remark 1. The basis functions with double knots are still nonnegative although the values of the local shape parameters do not satisfy inequalities in (4) and (6). We take $N_{I, i_{0}-1}(u)$ in (9), for example:

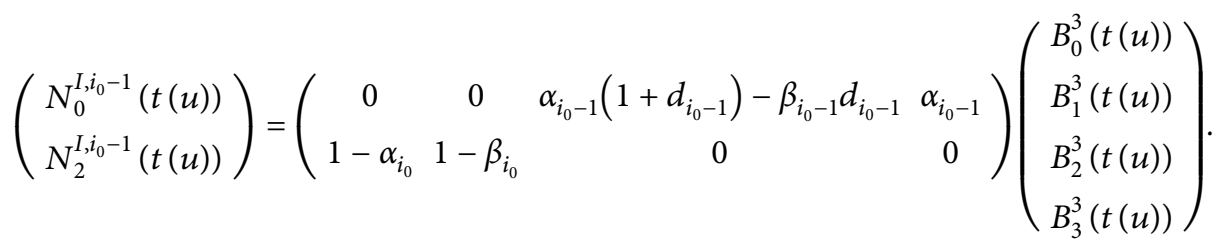

Considering $\alpha_{i_{0}-1}=1, \alpha_{i_{0}}=0$ and $d_{i_{0}-1}=0$, we can see that $N_{I, i_{0}-1}(u)$ with a local shape parameter $\beta_{i_{0}}$ is nonnegative.

We display the basis functions $\left\{N_{e, i}(u)\right\}_{i=-\infty}^{+\infty}(e=\mathrm{I}, \mathrm{II})$ with double knots in Figures 2 and 3.

\section{Spline Curve}

3.1. Definition. Given a set of control points $\left\{\mathbf{P}_{i}\right\}_{i=1}^{n}$ and a nonuniform knot sequence $\left\{u_{i}\right\}_{i=-\infty}^{+\infty}$, two kinds of nonuniform piecewise polynomial spline curves with local shape parameter series are defined as follows.

Definition 2. Given a set of control points $\left\{\mathbf{P}_{i}\right\}_{i=1}^{n}$ and a nonuniform knot sequence $\left\{u_{i}\right\}_{i=1}^{n+3}$, the $\mathrm{W}^{3} \mathrm{D}^{3} \mathrm{C}^{1} \mathrm{P}^{2}$ spline curve, the $\mathrm{W}^{3} \mathrm{D}^{4} \mathrm{C}^{2} \mathrm{P}^{1}$ spline curve $\mathbf{P}_{e}(u)(e=\mathrm{I}, \mathrm{II})$, and the corresponding $i$ th $(3 \leq i \leq n)$ curve segment is defined as follows:

$$
\begin{aligned}
\mathbf{P}_{e}(u) & =\sum_{i=1}^{n} N_{e, i}(u) \mathbf{P}_{i}, \quad u \in\left[u_{3}, u_{n+1}\right],(e=\mathrm{I}, \mathrm{II}), \\
\mathbf{P}_{e, i}(u) & =N_{2}^{e, i-2}(t(u)) \mathbf{P}_{i-2}+N_{1}^{e, i-1}(t(u)) \mathbf{P}_{i-1}+N_{0}^{e, i}(t(u)) \mathbf{P}_{i}, \quad u \in\left[u_{i}, u_{i+1}\right),(e=\mathrm{I}, \mathrm{II}) .
\end{aligned}
$$




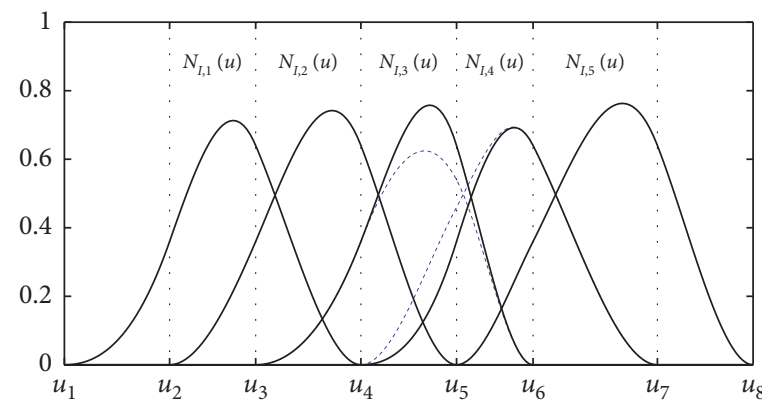

(a)

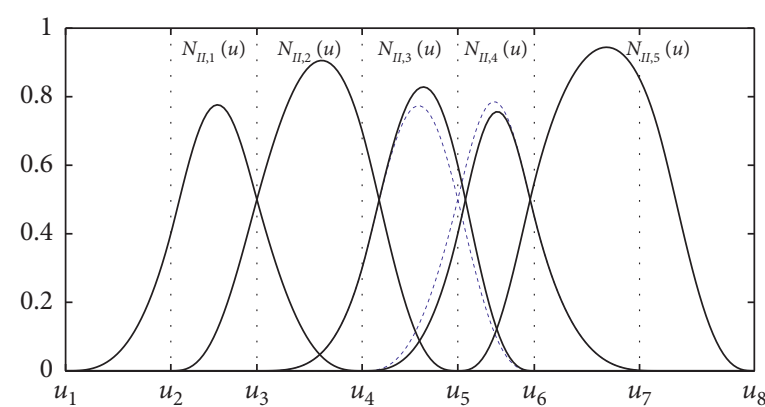

(b)

Figure 1: (a) The $W^{3} D^{3} C^{1} P^{2}$ basis functions $\left\{N_{\mathrm{I}, i}(u)\right\}$ with different $\alpha_{4}$; (b) The $W^{3} D^{4} C^{2} P^{1}$ basis functions $\left\{N_{\mathrm{II}, i}(u)\right\}$ with different $\gamma_{4}$.

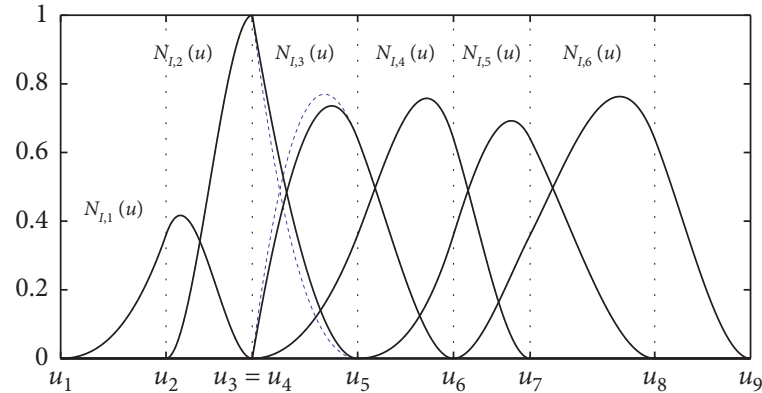

(a)

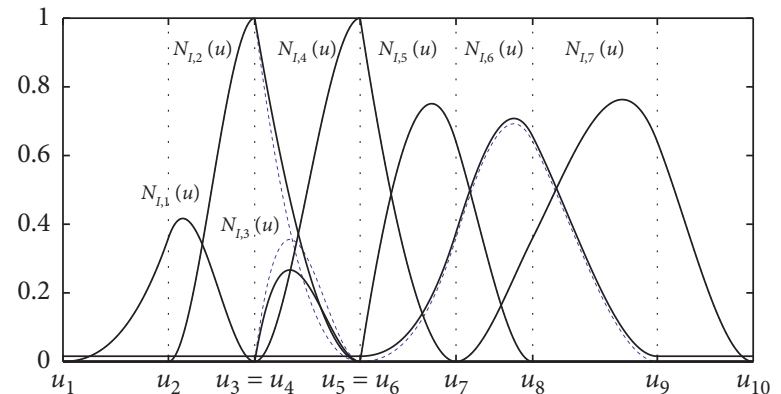

(b)

Figure 2: The $W^{3} D^{3} C^{1} P^{2}$ basis functions $\left\{N_{I, i}(u)\right\}$ with different $\beta^{3}$ (a) double knots and (b) consecutive double knots.

We plot the spline curve $\mathbf{P}_{e}(u)(e=\mathrm{I}$, II) in Figures 4 and 5 .

3.2. Properties. The properties of the $W^{3} D^{3} C^{1} P^{2}$ spline curve $\mathbf{P}_{\mathrm{I}}(u)$ and the $W^{3} D^{4} C^{2} P^{1}$ spline curve $\mathbf{P}_{\mathrm{II}}(u)$ are already shown in this section.

Property 9 (local shape adjustability). When the $i$ th shape parameter is varying, such as $\alpha_{i}, \beta_{i}$, or $\gamma_{i}$, only two curve segments $\mathbf{P}_{e, i}(u)$ and $\mathbf{P}_{e, i+1}(u)$ in $\mathbf{P}_{e}(u)(e=\mathrm{I}, \mathrm{II})$ will be changed.

Proof. Here, we take $\alpha_{i}$ in $\mathbf{P}_{I, i}(u)$ as example. Based on the definition of $\mathbf{P}_{I, i}(u)$ in (9), the matrix form of the curve segment $\mathbf{P}_{I, i}(u)$ is written as follows:

$$
\begin{aligned}
\mathbf{P}_{I, i}(u) & =\left(\mathbf{P}_{i-2}, \mathbf{P}_{i-1}, \mathbf{P}_{i}\right)\left(\begin{array}{c}
N_{2}^{I, i-2}(t(u)) \\
N_{1}^{I, i-1}(t(u)) \\
N_{0}^{I, i}(t(u))
\end{array}\right), \\
& =\left(\mathbf{P}_{i-2}, \mathbf{P}_{i-1}, \mathbf{P}_{i}\right)\left(\begin{array}{cccc}
1-\alpha_{i-1} & 1-\beta_{i-1} & 0 & 0 \\
\alpha_{i-1} & \beta_{i-1} & 1-\alpha_{i}\left(1+d_{i}\right)+\beta_{i} d_{i} & 1-\alpha_{i} \\
0 & 0 & \alpha_{i}\left(1+d_{i}\right)-\beta_{i} d_{i} & \alpha_{i}
\end{array}\right)\left(\begin{array}{c}
B_{0}^{3}(t(u)) \\
B_{1}^{3}(t(u)) \\
B_{2}^{3}(t(u)) \\
B_{3}^{3}(t(u))
\end{array}\right) .
\end{aligned}
$$

Hence, the curve segment $\mathbf{P}_{I, i}(u)$ can be adjusted by varying the value of $\alpha_{i-1}$ and $\alpha_{i}$. And, we also can obtain that $\mathbf{P}_{I, i+1}(u)$ can be adjusted by varying the value of $\alpha_{i}$ and $\alpha_{i+1}$. That is to say, only $\mathbf{P}_{I, i}(u)$ and $\mathbf{P}_{I, i+1}(u)$ will modify when $\alpha_{i}$ changes.

The rest of Property 9 can be proved similarly.
Property 10 (geometric invariance). The spline curves $\mathbf{P}_{e}(u)(e=\mathrm{I}$, II) only rely on the control points, whereas they are unrelated to the coordinate system.

Property 11 (affine invariance). Barycentric combinations (7) are invariant under affine maps. 


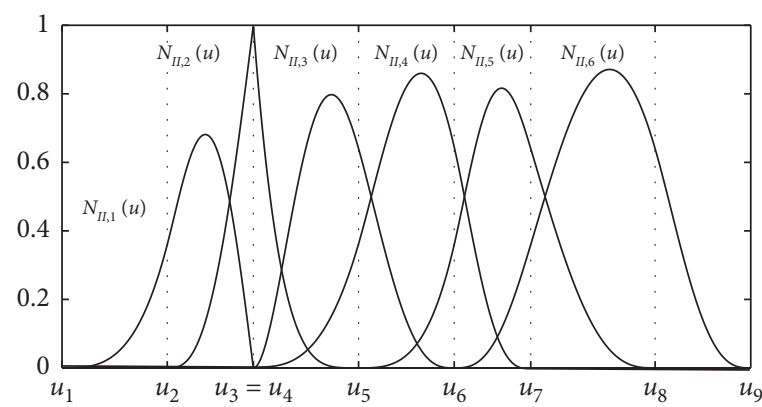

(a)

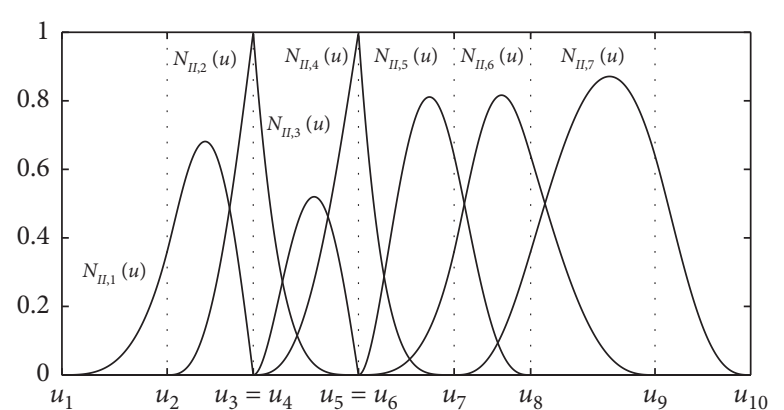

(b)

FIgURE 3: The $W^{3} D^{4} C^{2} P^{1}$ basis functions $\left\{N_{\mathrm{II}, i}(u)\right\}$ with (a) double knots and (b) consecutive double knots.

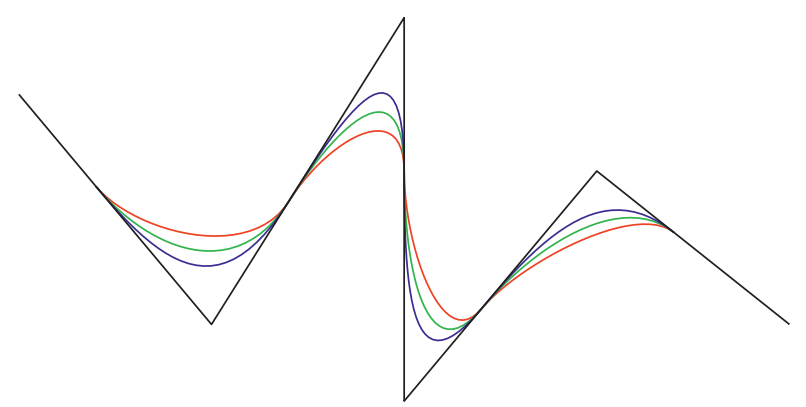

(a)

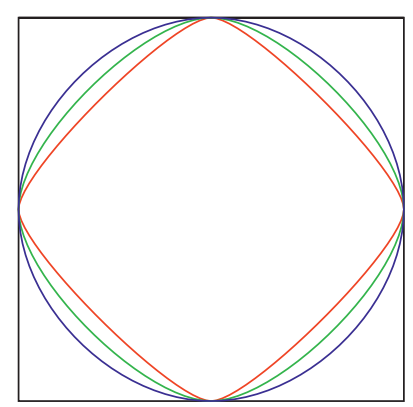

(b)

FIgURE 4: The $W^{3} D^{3} C^{1} P^{2}$ spline curve $(P)_{\mathrm{I}}(u)$ with different shape parameter series: (a) open curves; (b) closed curves.

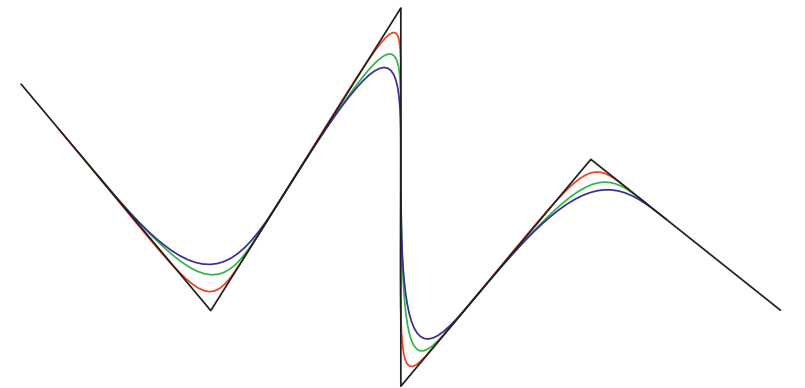

(a)

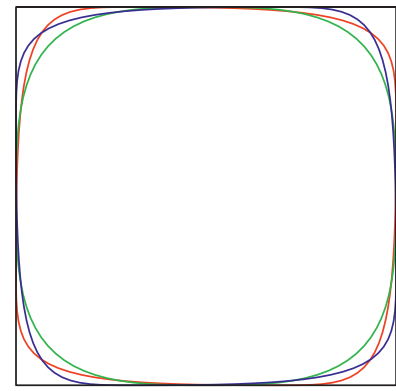

(b)

Figure 5: The $W^{3} D^{4} C^{2} P^{1}$ spline curve $(P)_{\text {II }}(u)$ with different shape parameter series: (a) open curves; (b) closed curves.

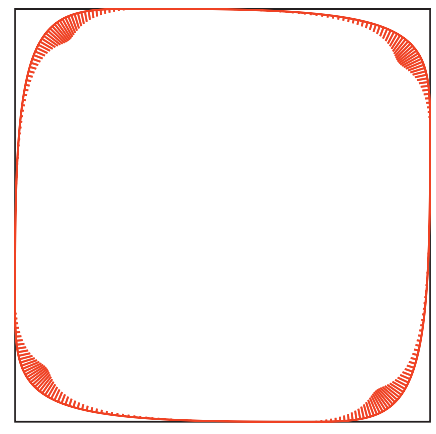

(a)

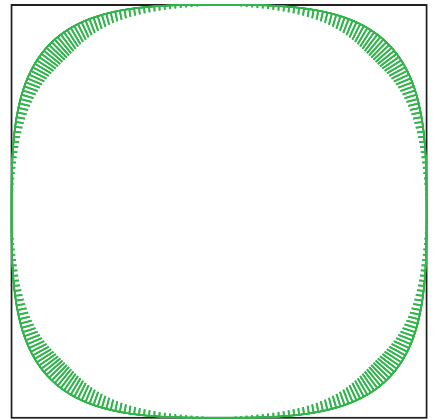

(b)

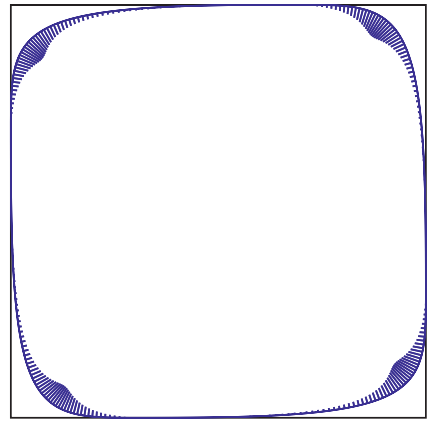

(c)

FIgURE 6: The curvature distributions of the $W^{3} D^{4} C^{2} P^{1}$ spline curve with different shape parameter series. 
Property 12 (convex hull). The curve segment $\mathbf{P}_{e}(u)(e=$ I, II) is located in the convex hull of the control points $\left\{\mathbf{P}_{j}\right\}_{j=i-2}^{i}$.

Property 13 (continuity). The $W^{3} D^{3} C^{1} P^{2}$ spline curve $\mathbf{P}_{I}(u)$ is $C^{1}$ continuous, and the $W^{3} D^{4} C^{2} P^{1}$ spline curve $\mathbf{P}_{\mathrm{II}}(u)$ is $C^{2}$ continuous.

Remark 2. The curvature of $W^{3} D^{4} C^{2} P^{1}$ spline curve is continuous based on its $C^{2}$ continuity. We take the $W^{3} D^{4} C^{2} P^{1}$ spline curves in Figure 5(b), for example.

It is obvious that different shape parameter series bring different curvature distributions in Figure 6. How to get the most stable curvature? That is a challenge in our future work.

Property 14 (degeneracy). If $\alpha_{i}=\left(d_{i} / 1+d_{i}\right), \beta_{i}=\left(d_{i}+\right.$ $\left.(2 / 3) / 1+d_{i}\right)$, the $\mathrm{W}^{3} \mathrm{D}^{3} \mathrm{C}^{1} \mathrm{P}^{2}$ spline curve $\mathbf{P}_{\mathrm{I}}(u)$ degenerates into the quadratic nonuniform $B$-spline curve.

Property 15 (shape-preserving property). The $\mathrm{W}^{3} \mathrm{D}^{3} \mathrm{C}^{1} \mathrm{P}^{2}$ spline curve $\mathbf{P}_{\mathrm{I}}(u)$ is shape-preserving if (13) holds; the $W^{3} D^{4} C^{2} P^{1}$ spline curve $\mathbf{P}_{\mathrm{II}}(u)$ is shape-preserving if (14) holds.

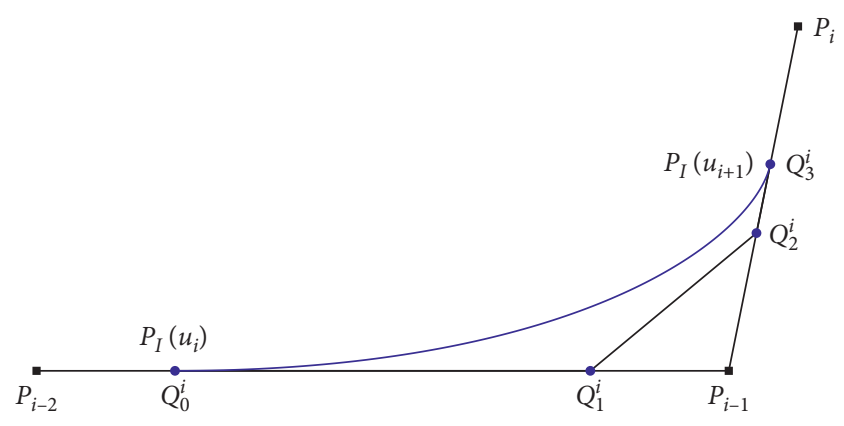

Figure 7: The Bézier representation of the curve segment $\mathrm{P}_{\mathrm{I}, \mathrm{i}}(\mathrm{u})$.

$$
\begin{array}{r}
\alpha_{i}<\beta_{i} \\
\left(1-d_{i}^{2}\right) \gamma_{i}+d_{i}^{2}>0 .
\end{array}
$$

Proof. According to the matrix form of the curve segment (12), $\mathbf{P}_{I, i}(u)$ is the cubic Bézier curve with control points $\left\{\mathbf{Q}_{j}^{i}\right\}_{j=0}^{3}$ as follows:

$$
\left(\mathbf{Q}_{0}^{i}, \mathbf{Q}_{1}^{i}, \mathbf{Q}_{2}^{i}, \mathbf{Q}_{3}^{i}\right)=\left(\mathbf{P}_{i-2}, \mathbf{P}_{i-1}, \mathbf{P}_{i}\right)\left(\begin{array}{cccc}
1-\alpha_{i-1} & 1-\beta_{i-1} & 0 & 0 \\
\alpha_{i-1} & \beta_{i-1} & 1-\alpha_{i}\left(1+d_{i}\right)+\beta_{i} d_{i} & 1-\alpha_{i} \\
0 & 0 & \alpha_{i}\left(1+d_{i}\right)-\beta_{i} d_{i} & \alpha_{i}
\end{array}\right)
$$

We take $\mathbf{Q}_{0}^{i}$, for example:

$$
\mathbf{Q}_{0}^{i}=\left(1-\alpha_{i-1}\right) \mathbf{P}_{i-2}+\alpha_{i-1} \mathbf{P}_{i-1} .
$$

Thus, $\mathbf{Q}_{0}^{i}$ is the linear combination of $\mathbf{P}_{i-2}$ and $\mathbf{P}_{i-1}$ and lies on the line segment $\mathbf{P}_{i-2} \mathbf{P}_{i-1} \cdot \mathbf{Q}_{1}^{i}, \mathbf{Q}_{2}^{i}$, and $\mathbf{Q}_{3}^{i}$ are similar to $\mathbf{Q}_{0}^{i}$. When inequality (13) holds, the order of these points is $\quad \mathbf{P}_{i-2} \longrightarrow \mathbf{Q}_{0}^{i} \longrightarrow \mathbf{Q}_{1}^{i} \longrightarrow \mathbf{P}_{i-1} \longrightarrow \mathbf{Q}_{2}^{i} \longrightarrow \mathbf{Q}_{3}^{i} \longrightarrow \mathbf{P}_{i}$ (Figure 7). Meanwhile, if the control polygon $\mathbf{P}_{i-2} \mathbf{P}_{i-1} \mathbf{P}_{i}$ is monotone or convex, the control polygon $\mathbf{Q}_{0}^{i} \mathbf{Q}_{1}^{i} \mathbf{Q}_{2}^{i} \mathbf{Q}_{3}^{i}$ is also monotone or convex. So, the curve segment $\mathbf{P}_{\mathrm{I}, i}(u)$ considered as a cubic Bézier curve is monotone or convex, too. The shape-preserving property of the $\mathrm{W}^{3} \mathrm{D}^{3} \mathrm{C}^{1} \mathrm{P}^{2}$ spline curve $\mathbf{P}_{\mathrm{I}}(u)$ can be obtained thus. Similarly, the rest of Property 15 about the $W^{3} D^{4} C^{2} P^{1}$ spline curve $\mathbf{P}_{\mathrm{II}}(u)$ also can be proved.
Property 16 (variation diminishing property). The $W^{3} D^{3} C^{1} P^{2}$ spline curve $\mathbf{P}_{\mathrm{I}}(u)$ is variation diminishing if (13) holds; the $W^{3} D^{4} C^{2} P^{1}$ spline curve $\mathbf{P}_{\mathrm{II}}(u)$ is variation diminishing if (14) holds.

Proof. It is a direct application of the Bézier representation of $\mathbf{P}_{e, i}(u)(e=\mathrm{I}, \mathrm{II})$.

Remark 3. The properties of shape-preserving and variation diminishing are very important for the spline curve. Therefore, when we construct the $W^{3} D^{3} C^{1} P^{2}$ spline curve and the $W^{3} D^{4} C^{2} P^{1}$ spline curve, we choose the shape parameter series based on not only (4) and (6) but also (13) and (14). 
3.3. Geometric Effect of the Shape Parameters. From Property 9 , we can adjust the $W^{3} D^{3} C^{1} P^{2}$ spline curve segment $\mathbf{P}_{I, i}(u)$ by altering the value of $\alpha_{i-1}, \alpha_{i}, \beta_{i-1}$, and $\beta_{i}$ and adjust the $W^{3} D^{4} C^{2} P^{1}$ spline curve segment $\mathbf{P}_{\mathrm{II}, i}(u)$ by altering the value of $\gamma_{i-1}$ and $\gamma_{i}$. We present the effect of the shape parameters in the shape modification by the derivatives as follows:

$$
\begin{aligned}
\frac{\partial}{\partial \alpha_{i}} \mathbf{P}_{I, i}(u) & =\left(\left(1+d_{i}\right) B_{2}^{3}(t(u))+B_{3}^{3}(t(u))\right)\left(\mathbf{P}_{i}-\mathbf{P}_{i-1}\right), \\
\frac{\partial}{\partial \alpha_{i-1}} \mathbf{P}_{I, i}(u) & =B_{0}^{3}(t(u))\left(\mathbf{P}_{i-1}-\mathbf{P}_{i-2}\right), \\
\frac{\partial}{\partial \beta_{i}} \mathbf{P}_{I, i}(u) & =d_{i} B_{2}^{3}(t(u))\left(\mathbf{P}_{i-1}-\mathbf{P}_{i}\right), \\
\frac{\partial}{\partial \beta_{i-1}} \mathbf{P}_{I, i}(u) & =B_{1}^{3}(t(u))\left(\mathbf{P}_{i-1}-\mathbf{P}_{i-2}\right), \\
\frac{\partial}{\partial \gamma_{i}} \mathbf{P}_{I I, i}(u) & =\left(\frac{1}{2}\left(1+d_{i}\right) B_{3}^{4}(t(u))+B_{4}^{4}(t(u))\right)\left(\mathbf{P}_{i}-\mathbf{P}_{i-1}\right), \\
\frac{\partial}{\partial \gamma_{i-1}} \mathbf{P}_{I I, i}(u) & =\left(B_{0}^{4}(t(u))+\frac{1}{2} \frac{\left(1+d_{i-1}\right)}{d_{i-1}} B_{1}^{4}(t(u))\right)\left(\mathbf{P}_{i-1}-\mathbf{P}_{i-2}\right) .
\end{aligned}
$$

These 6 derivatives are similar; hence, we take (17) as example to explain the geometric effect of the shape parameters $\alpha_{i}$ with respect to the curve segment $\mathbf{P}_{\mathrm{I}, i}(u)$. When we increase $\alpha_{i}$ only while fixing $\alpha_{i-1}, \beta_{i-1}, \beta_{i}$, the curve segment $\mathbf{P}_{\mathrm{I}, i}(u)$ will be away from $\mathbf{P}_{i-1}$ and closed to $\mathbf{P}_{i}$. Figure 8 shows the geometric effect of $\alpha_{i}, \beta_{i}$, and $\gamma_{i}$ from (17)-(19).

Based on the shape parameters, the two extensions are more flexible than the quadratic $B$-spline curve. We show that the $W^{3} D^{3} C^{1} P^{2}$ spline curve and the $W^{3} D^{4} C^{2} P^{1}$ spline curve can be closer to or more far away from the given control polygon than the quadratic $B$-spline curves with appropriate shape parameters in Figure 9.

\section{Interpolation}

4.1. The Geometric Significance of the Shape Parameter. Before we apply the two extensions of the quadratic nonuniform $B$-spline curve to interpolation, we present the position and the corresponding tangent direction of them at $u=u_{i+1}$. It follows from a routine computation that

$$
\begin{aligned}
\mathbf{P}_{\mathrm{I}}\left(u_{i+1}\right) & =\left(1-\alpha_{i}\right) \mathbf{P}_{i-1}+\alpha_{i} \mathbf{P}_{i}, \\
\mathbf{P}_{\mathrm{I}}^{\prime}\left(u_{i+1}\right) & =\frac{3}{h_{i+1}}\left(\beta_{i}-\alpha_{i}\right)\left(\mathbf{P}_{i}-\mathbf{P}_{i-1}\right), \\
\mathbf{P}_{\mathrm{II}}\left(u_{i+1}\right) & =\left(1-\gamma_{i}\right) \mathbf{P}_{i-1}+\gamma_{i} \mathbf{P}_{i}, \\
\mathbf{P}_{\mathrm{II}}^{\prime}\left(u_{i+1}\right) & =\frac{2}{h_{i+1}} \frac{\gamma_{i}+d_{i}^{2}\left(1-\gamma_{i}\right)}{d_{i}\left(1+d_{i}\right)}\left(\mathbf{P}_{i}-\mathbf{P}_{i-1}\right) .
\end{aligned}
$$

Consequently, the point $\mathbf{P}_{e}\left(u_{i+1}\right)(e=\mathrm{I}, \mathrm{II})$ lies on the line segment $\mathbf{P}_{i-1} \mathbf{P}_{i}$, and the tangent direction at $\mathbf{P}_{e}\left(u_{i+1}\right)(e=\mathrm{I}, \mathrm{II})$ is parallel to $\mathbf{P}_{i-1} \mathbf{P}_{i}$ from (20) and (21).
Furthermore, we can obtain the geometric significance of the shape parameter as follows:

$$
\begin{aligned}
& \alpha_{i}=\frac{\left\|\mathbf{P}_{i-1} \mathbf{P}_{I}\left(u_{i+1}\right)\right\|}{\left\|\mathbf{P}_{i-1} \mathbf{P}_{i}\right\|}, \\
& \gamma_{i}=\frac{\left\|\mathbf{P}_{i-1} \mathbf{P}_{\mathrm{II}}\left(u_{i+1}\right)\right\|}{\left\|\mathbf{P}_{i-1} \mathbf{P}_{i}\right\|} .
\end{aligned}
$$

Figures 8(a), 8(c), and Figure 9 show it intuitively.

Based on the geometric significance of the shape parameters (20) and (21), two interpolations are presented as follows.

4.2. Interpolation of the Position and Tangent Direction. Given a set of points $\left\{\mathbf{Q}_{i}\right\}_{i=1}^{n}$ and the unit direct vectors $\left\{\mathbf{T}_{i}\right\}_{i=1}^{n}$ on them as the tangent directions, we can construct a $W^{3} D^{3} C^{1} P^{2}$ spline curve $\mathbf{P}_{I}(u)\left(u \in\left[u_{3}, u_{n+1}\right]\right)$ from "IPT (Interpolation of the Position and Tangent direction) algorithm," without solving a system of linear functions such that

$$
\begin{array}{r}
\mathbf{P}_{I}\left(u_{i+2}\right)=\mathbf{Q}_{i}, \\
\mathbf{P}_{\mathrm{I}}^{\prime}\left(u_{i+2}\right) \| \mathbf{T}_{i}
\end{array}
$$

\subsubsection{IPT Algorithm}

Step 1. We construct the control polygon $\mathbf{P}_{1} \mathbf{P}_{2}, \ldots, \mathbf{P}_{n} \mathbf{P}_{n+1}$ first, such that $\mathbf{Q}_{i}$ lies on the line segment $\mathbf{P}_{i} \mathbf{P}_{i+1}$ and $\mathbf{T}_{i} \| \mathbf{P}_{i} \mathbf{P}_{i+1}$.

Step 2. We set the local shape parameter series $\left\{\alpha_{i}\right\}_{i=2}^{n+1}$ from (22) as 


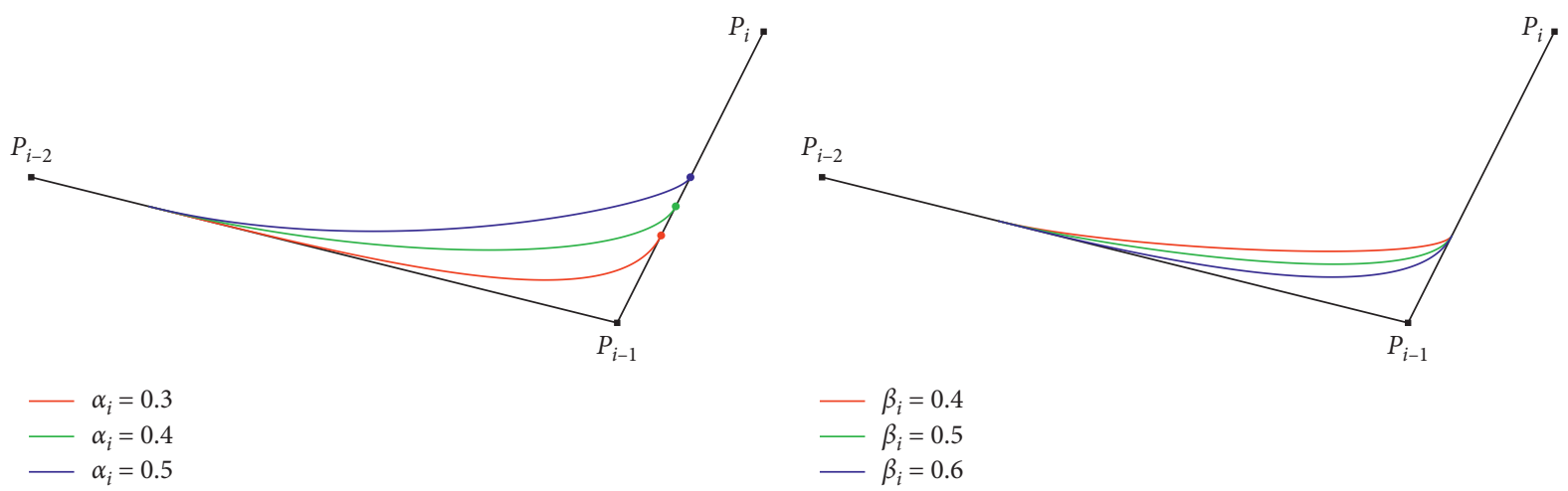

(a)

(b)

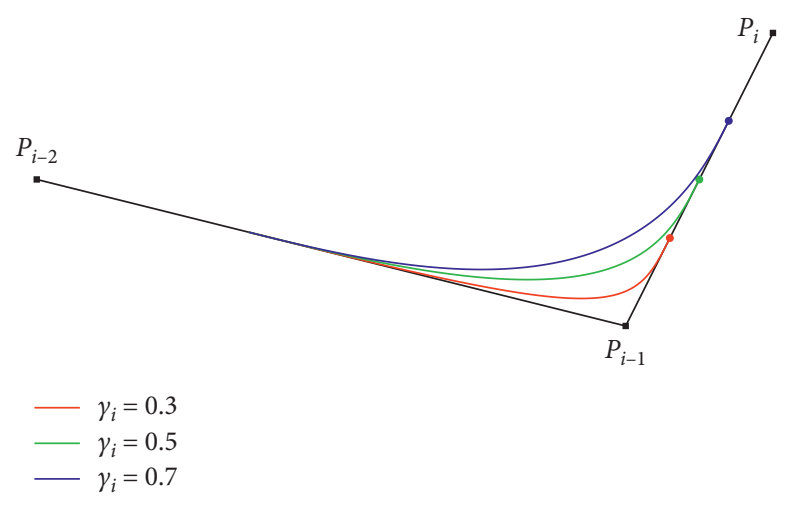

(c)

Figure 8: The curve segments $\mathbf{P}_{e, i}(u)(e=\mathrm{I}$, II $)$ on the interval $\left[u_{i}, u_{i+1}\right]$ with different shape parameters: $(\mathrm{a})(P)_{I, i}(u)$ is away from the $(P)_{i-1}$ with the increase in $\alpha_{i} ;(\mathrm{b})(P)_{\mathrm{I}, i}(u)$ is closed to the $(P)_{i-1}$ with the increase in $\beta_{i} ;(\mathrm{c})(P)_{\mathrm{II}, i}(u)$ is away from the $(P)_{i-1}$ with the increase in $\gamma_{i}$.

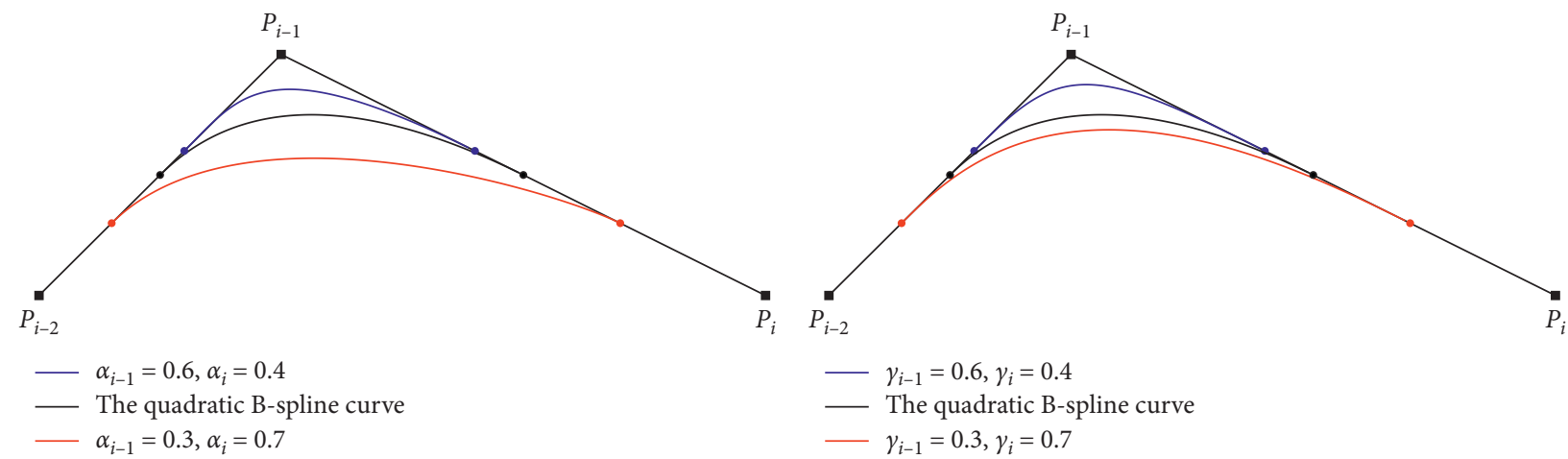

(a)

(b)

Figure 9: The two extensions can be closer to or more far away from the control polygon than the $B$-spline curves: (a) the $W^{3} D^{3} C^{1} P^{2}$ spline curve segment $P_{\mathrm{I}, \mathrm{i}}(u)$; (b) the $W^{3} D^{4} C^{2} P^{1}$ spline curve segment $P_{\mathrm{II}, i}(u)$.

$$
\alpha_{i}=\frac{\left\|\mathbf{P}_{i-1} \mathbf{Q}_{i-1}\right\|}{\left\|\mathbf{P}_{i-1} \mathbf{P}_{i}\right\|}, \quad i=2,3, \ldots, n+1
$$

Step 3. We select the arbitrary local shape parameter series $\left\{\beta_{i}\right\}_{i=2}^{n+1}$ from the scopes in (4) and (13) based on $\left\{\alpha_{i}\right\}_{i=2}^{n+1}$ above.

Remark 3. We can set the first control point $\mathbf{P}_{1}$ and the last control point $\mathbf{P}_{n+1}$ as follows:

$$
\begin{aligned}
& \left\|\mathbf{P}_{1} \mathbf{Q}_{1}\right\|=\left\|\mathbf{Q}_{1} \mathbf{P}_{2}\right\|, \\
& \left\|\mathbf{P}_{n} \mathbf{Q}_{n}\right\|=\left\|\mathbf{Q}_{n} \mathbf{P}_{n+1}\right\| .
\end{aligned}
$$

The corresponding shape parameters $\alpha_{2}=\alpha_{n+1}=0.5$. 
Comparing (24) with (22), we can see that the $\mathrm{W}^{3} \mathrm{D}^{3} \mathrm{C}^{1} \mathrm{P}^{2}$ spline curve $\mathbf{P}_{\mathrm{I}}(u)$ from the "IPT algorithm" interpolates both the position $\left\{\mathbf{Q}_{i}\right\}_{i=1}^{n}$ and the corresponding tangent direction $\left\{\mathbf{T}_{i}\right\}_{i=1}^{n}$ with local shape parameter series $\left\{\beta_{i}\right\}_{i=2}^{n+1}$ (Figure 10).

Unfortunately, the $W^{3} D^{3} C^{1} P^{2}$ spline curve $\mathbf{P}_{\mathrm{II}}(u)$ is not suitable to interpolate the position and tangent direction. When we set the local shape parameter series $\left\{\gamma_{i}\right\}_{i=-\infty}^{+\infty}$ based on (22) like (24), the value of shape parameter $\gamma_{i}$ may be against the scope in (6), which keeps the nonnegativity of the basis function $\left\{N_{I I, i}(u)\right\}_{i=-\infty}^{+\infty}$.

4.3. Interpolation of a Line Segment. Given a line segment $\mathbf{A B}$, we can construct a $\mathrm{W}^{3} \mathrm{D}^{3} \mathrm{C}^{1} \mathrm{P}^{2}$ spline curve $\mathbf{P}_{I}(u)$ as follows, such that the curve segment $\mathbf{P}_{I, i}(u)\left(u \in\left[u_{i}, u_{i+1}\right]\right)$ is the line segment $\mathbf{A B}$.

We set $\mathbf{P}_{i-2}, \mathbf{A}, \mathbf{P}_{i-1}, \mathbf{B}$, and $\mathbf{P}_{i}$ as collinear. $\mathbf{A}$ is between $\mathbf{P}_{i-2}$ and $\mathbf{P}_{i-1}$, and $\mathbf{B}$ is between $\mathbf{P}_{i-1}$ and $\mathbf{P}_{i}$ (Figure 11). Then, we set the corresponding shape parameter from (18) as follows:

$$
\begin{gathered}
\alpha_{i-1}=\frac{\left\|\mathbf{P}_{i-2} \mathbf{A}\right\|}{\left\|\mathbf{P}_{i-2} \mathbf{P}_{i-1}\right\|}, \\
\alpha_{i}=\frac{\left\|\mathbf{P}_{i-1} \mathbf{B}\right\|}{\left\|\mathbf{P}_{i-1} \mathbf{P}_{i}\right\|} .
\end{gathered}
$$

Hence, (26) gives

$$
\begin{aligned}
\mathbf{P}_{I, i}\left(u_{i}\right) & =\mathbf{A}, \\
\mathbf{P}_{I, i}\left(u_{i+1}\right) & =\mathbf{B} .
\end{aligned}
$$

In view of the convex hull property, the curve segment $\mathbf{P}_{\mathrm{I}, i}(u)$ is in the convex hull of $\left\{\mathbf{P}_{j}\right\}_{j=i-2}^{i}$, which is a line segment itself. The local shape parameter series $\left\{\beta_{i}\right\}_{i=2}^{n+1}$ can be selected freely from the scopes in (4) and (13). Hence, the curve segment $\mathbf{P}_{\mathrm{I}, i}(u)$ is the line segment $\mathbf{A B}$. Note that the whole spline curve $\mathbf{P}_{\mathrm{I}}(u)$ is still $\mathrm{C}^{1}$ continuous.

Similarly, we can get a $\mathrm{W}^{3} \mathrm{D}^{4} \mathrm{C}^{2} \mathrm{P}^{1}$ spline curve $\mathbf{P}_{I I}(u)$ in which the curve segment $\mathbf{P}_{\mathrm{II}, i}(u)\left(u \in\left[u_{i}, u_{i+1}\right]\right)$ is the line segment $\mathbf{A B}$, too. Note that the whole spline curve $\mathbf{P}_{\mathrm{II}}(u)$ is still $C^{2}$ continuous. From (22), the corresponding shape parameters are set as follows:

$$
\begin{gathered}
\gamma_{i-1}=\frac{\left\|\mathbf{P}_{i-2} \mathbf{A}\right\|}{\left\|\mathbf{P}_{i-2} \mathbf{P}_{i-1}\right\|}, \\
\gamma_{i}=\frac{\left\|\mathbf{P}_{i-1} \mathbf{B}\right\|}{\left\|\mathbf{P}_{i-1} \mathbf{P}_{i}\right\|} .
\end{gathered}
$$

Compared with $\mathbf{P}_{\mathrm{I}}(u)$, the corresponding shape parameters in $\mathbf{P}_{\mathrm{II}}(u)$ are fixed.

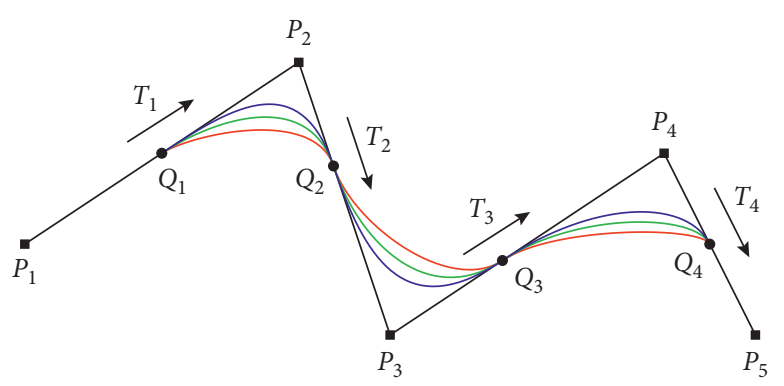

FIgure 10: The $W^{3} D^{3} C^{1} P^{2}$ spline curve $P_{\mathrm{I}}(u)$ interpolating the position $\left\{\mathbf{Q}_{i}\right\}_{i=1}^{4}$ and tangent direction $\left\{\mathbf{T}_{i}\right\}_{i=1}^{4}$ with different shape parameter series $\left\{\beta_{i}\right\}_{i=2}^{5}$

\section{Problems with Clamped Knot Vector}

In the B-spline model, clamped knot vector $\mathbf{U}=\left(u_{3}, u_{3}, u_{3}, u_{4}, \cdots, u_{n}, u_{n+1}, u_{n+1}, u_{n+1}\right)$ can be used to interpolate the first control point $\mathbf{P}_{1}$ and the last control point $\mathbf{P}_{n}$. The corresponding tangent direction at $\mathbf{P}_{1}$ or $\mathbf{P}_{n}$ is parallel to $\mathbf{P}_{1} \mathbf{P}_{2}$ or $\mathbf{P}_{n-1} \mathbf{P}_{n}$, respectively. Clamped knot vector is also suitable for the new basis functions constructed in Section 2.3.

With a clamped knot vector $\mathbf{U}$, we can set $\alpha_{1}=\alpha_{n+1}=$ 1 and $\alpha_{2}=\alpha_{n+2}=0$ for the $W^{3} D^{3} C^{1} P^{2}$ spline curve $\mathbf{P}_{I}(u)$ as Property 7 , and we have

$$
\begin{aligned}
& \mathbf{P}_{\mathrm{I}}\left(u_{3}\right)=\mathbf{P}_{1}, \\
& \mathbf{P}_{\mathrm{I}}\left(u_{n}\right)=\mathbf{P}_{n}, \\
& \mathbf{P}_{\mathrm{I}}^{\prime}\left(u_{3}^{+}\right) \| \mathbf{P}_{1} \mathbf{P}_{2}, \\
& \mathbf{P}_{\mathrm{I}}^{\prime}\left(u_{n}^{-}\right)=\mathbf{0} .
\end{aligned}
$$

In Figure 12(a), with a clamped knot vector, we can see that $\mathbf{P}_{I}(u)$ interpolates the first control point $\mathbf{P}_{1}$ and the last control point $\mathbf{P}_{n}$, and the tangential directs at $\mathbf{P}_{1}$ is parallel to $\mathbf{P}_{1} \mathbf{P}_{2}$. However, the tangent direction at $\mathbf{P}_{n}$ is not parallel to $\mathbf{P}_{n-1} \mathbf{P}_{n}$ based on (29). In order to make the tangent direction at $\mathbf{P}_{n}$ is parallel to $\mathbf{P}_{n-1} \mathbf{P}_{n}$, we can construct $\mathbf{P}_{I}(u)$ as follows:

Step 1. Unclamp the right end of the knot vector $\mathbf{U}$ as $\widetilde{U}=\left(u_{3}, u_{3}, u_{3}, u_{4}, \ldots, u_{n}, u_{n+1}, u_{n+2}, u_{n+3}\right)$.

Step 2. Choose the auxiliary end control point $\widetilde{P}_{n}$ on the extension line of $\mathbf{P}_{n-1} \mathbf{P}_{n}$, which takes the place of $\mathbf{P}_{n}$. Step 3. Set the shape parameter $\alpha_{n+1}=\left\|\mathbf{P}_{n-1} \mathbf{P}_{n}\right\| /\left\|\mathbf{P}_{n-1} \widetilde{P}_{n}\right\|$ from (18). Hence, we have $\mathbf{P}_{I}\left(u_{n}\right)=\mathbf{P}_{n}$ and $\mathbf{P}_{\mathrm{I}}^{\prime}\left(u_{n}^{-}\right) \| \mathbf{P}_{n-1} \mathbf{P}_{n} \quad$ from $\quad$ (20) (Figure 12(b)).

$\mathbf{P}_{\mathrm{II}}(u)$ with a clamped knot vector also interpolates the first and the last control point, and the tangential direction at $\mathbf{P}_{n}$ is parallel to $\mathbf{P}_{n-1} \mathbf{P}_{n}$ (Figure 13(a)). However, the tangent direction at $\mathbf{P}_{1}$ is not parallel to $\mathbf{P}_{1} \mathbf{P}_{2}$. The corresponding construction of $\mathbf{P}_{\mathrm{II}}(u)$ is similar to the case of $\mathbf{P}_{\mathrm{I}}(u)$ (Figure 13(b)). 


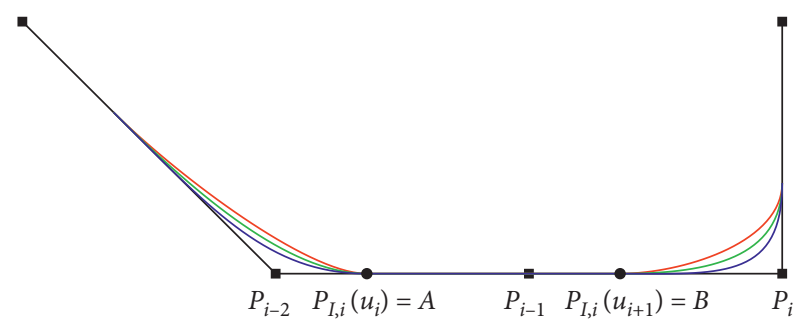

(a)

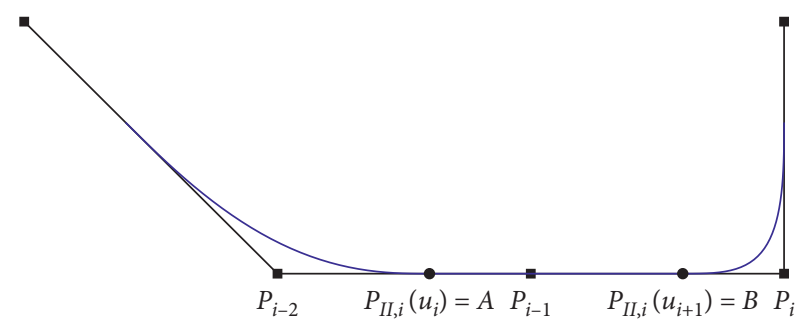

(b)

Figure 11: Interpolation of a line segment: (a) the $W^{3} D^{3} C^{1} P^{2}$ spline curve $P_{\mathrm{I}}(u)$; (b) the $W^{3} D^{4} C^{2} P^{1}$ spline curve $P_{\mathrm{II}}(u)$.

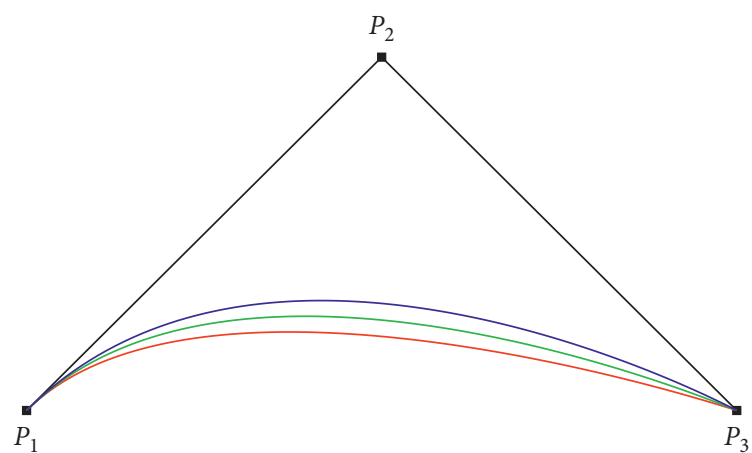

(a)

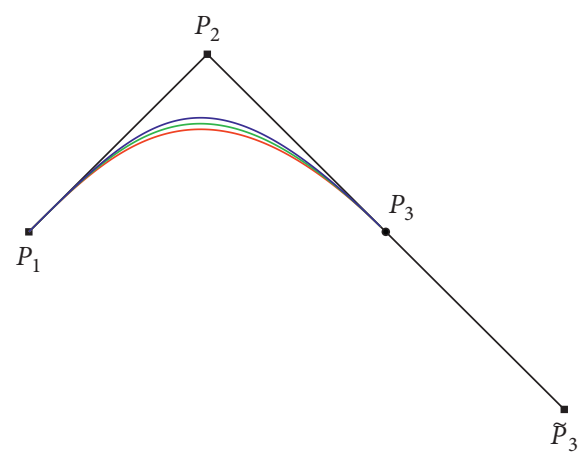

(b)

Figure 12: The improvement of the $W^{3} D^{3} C^{1} P^{2}$ spline curve $(P)_{\mathrm{I}}(u)$ : (a) knot vector $(0,0,0,1,1,1) ;(\mathrm{b})$ knot vector $(0,0,0,1,2,3)$ and control polygon $\mathbf{P}_{1} \mathbf{P}_{2} \widetilde{\mathbf{P}}_{3}$

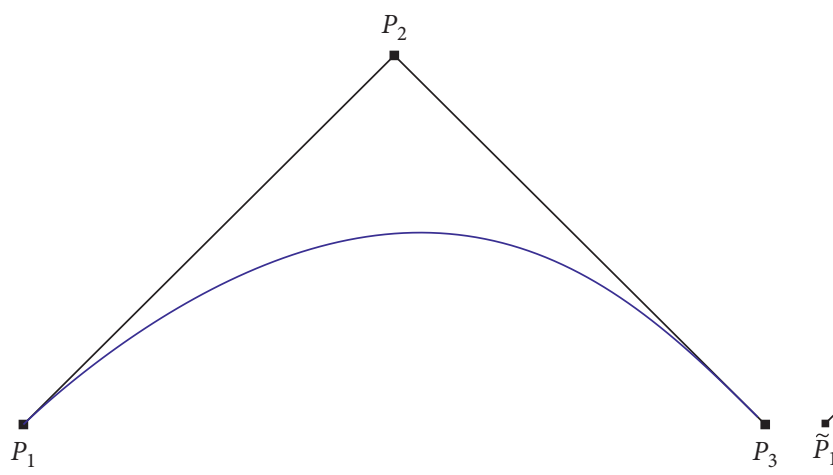

(a)

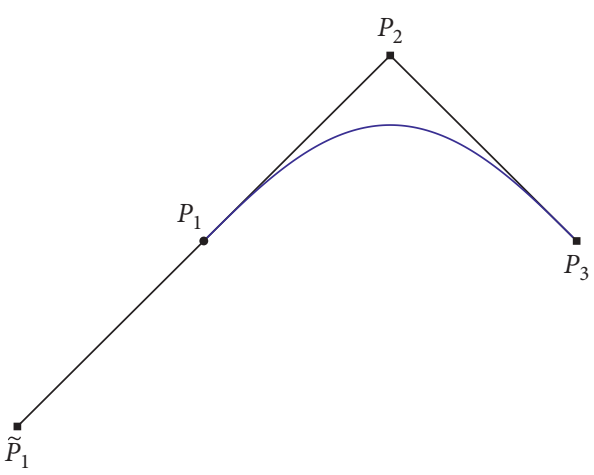

(b)

Figure 13: The improvement of the $W^{3} D^{4} C^{2} P^{1}$ spline curve $(P)_{\text {II }}(u)$ : (a) knot vector $(0,0,0,1,1,1)$; (b) knot vector $(-2,-1,0,1,2,3)$ and control polygon $\widetilde{\mathbf{P}}_{1} \mathbf{P}_{2} \mathbf{P}_{3}$.

TABle 2: Comparison between the quadratic nonuniform $B$-spline curve and the extensions.

\begin{tabular}{lccc}
\hline Properties & Quadratic nonuniform $B$-spline & $W^{3} D^{3} C^{1} P^{2}$ spline curve $P_{\mathrm{I}}(u)$ & $W^{3} D^{4} C^{2} P^{1}$ spline curve $P_{\mathrm{II}}(u)$ \\
\hline Geometric invariance & $\sqrt{ }$ & $\sqrt{ }$ & $\sqrt{ }$ \\
Affine invariance & $\sqrt{ }$ & $\sqrt{ }$ & $\sqrt{ }$ \\
Convex hull property & $\sqrt{ }$ & $\sqrt{ }$ & $\sqrt{ }$ \\
Shape-preserving property & $\sqrt{ }$ & $\sqrt{ }$ & $\sqrt{ }$ \\
Variation diminishing property & $\sqrt{ }$ & 3 & $\sqrt{ }$ \\
Width of the support intervals & 3 & 3 & 3 \\
Degree of polynomial basis functions & 2 & $C^{1}$ & 4 \\
Continuity of the basis functions & $C^{1}$ & 2 series: $\left\{\alpha_{i}\right\},\left\{\beta_{i}\right\}$ & $C^{2}$ \\
Number of local shape parameter series & None & & 1 series: $\left\{\gamma_{i}\right\}$ \\
\hline
\end{tabular}




\section{Conclusion}

Two extensions of the quadratic nonuniform $B$-spline curve, called the $W^{3} D^{3} C^{1} P^{2}$ spline curve and the $W^{3} D^{4} C^{2} P^{1}$ spline curve, are constructed in the paper. Both of the extensions have the same structure as the quadratic nonuniform $B$ spline curve. The properties of the extensions are similar with the ones of the quadratic nonuniform B-spline curves (Table 2). Particularly, the quadratic nonuniform $B$-spline curve is the special case of the $W^{3} D^{3} C^{1} P^{2}$ spline curve when taking specified shape parameter series. The shapes of the extensions can be adjusted by the shape parameter series locally even the control points fixed. The effects of varying the shape parameters on the extensions are illustrated. With the shape parameters, the extensions can move locally toward or against the control polygon. Based on the properties of the extensions, two applications, the interpolation of the position and tangent direction and interpolation of a line segment, are presented without solving a system of linear functions.

However, the extensions can not deal with the clamped knot vector very well. The tangent directions at the ends of control points are not parallel to the ends of control polygon. Although we construct the spline curve with the auxiliary points to avoid the defect, we will improve the basis functions in the case of multiple knots in our future research.

\section{Data Availability}

The data used to support the findings of this study are included within the article and are available from the corresponding author upon request.

\section{Conflicts of Interest}

The authors declare that they have no conflicts of interest.

\section{Acknowledgments}

This work was supported by the Natural Science Foundation of Zhejiang Province (nos. LY18F020026 and LY16F020020).

\section{References}

[1] L. Piegl and W. Tiller, The NURBS Book, Springer-Verlag, Berlin, Germany, 1997.

[2] L. Piegl, "Modifying the shape of rational $B$-splines. part 1: curves," Computer-Aided Design, vol. 21, no. 8, pp. 509-518, 1989.

[3] B. A. Barsky and J. C. Beatty, "Local control of bias and tension in beta-splines," ACM Transactions on Graphics, vol. 2, no. 2, pp. 109-134, 1983.

[4] Y. Lü, G. Wang, and X. Yang, "Uniform hyperbolic polynomial B-spline curves," Computer Aided Geometric Design, vol. 19, no. 6, pp. 379-393, 2002.

[5] M. Hoffmann, Y. Li, and G. Wang, "Paths of C-Bézier and C$B$-spline curves," Computer Aided Geometric Design, vol. 23, no. 5, pp. 463-475, 2006.

[6] S. Maqsood, M. Abbas, G. Hu et al., "A novel generalization of trigonometric bézier curve and surface with shape parameters and its applications," Mathematical Problems in Engineering, vol. 2020, Article ID 4036434, 25 pages, 2020.

[7] G. Hu, H. Li, M. Abbas et al., "Explicit continuity conditions for G1 connection of $S-\lambda$ curves and surfaces," Mathematics, vol. 8, p. 1359, 2020.

[8] A. Majeed, M. Abbas, F. Qayyum et al., "Geometric modeling using new cubic trigonometric B-spline functions with shape parameter," Mathematics, vol. 2020, no. 8, p. 2102, 2020.

[9] P. Costantini, "Curve and surface construction using variable degree polynomial splines," Computer Aided Geometric Design, vol. 17, no. 5, pp. 419-446, 2000.

[10] P. Costantini and C. Manni, "Geometric construction of spline curves with tension properties," Computer Aided Geometric Design, vol. 20, no. 8-9, pp. 579-599, 2003.

[11] K. F. Loe, “ $\alpha \mathrm{B}$-spline: a linear singular blending $B$-spline," The Visual Computer, vol. 12, no. 1, pp. 18-25, 1996.

[12] C.-L. Tai and G.-J. Wang, "Interpolation with slackness and continuity control and convexity-preservation using singular blending," Journal of Computational and Applied Mathematics, vol. 172, no. 2, pp. 337-361, 2004.

[13] X. Han, "Piecewise quartic polynomial curves with a local shape parameter," Journal of Computational and Applied Mathematics, vol. 195, no. 1-2, pp. 34-45, 2006.

[14] I. Juhász and M. Hoffmann, "On the quartic curve of Han," Journal of Computational and Applied Mathematics, vol. 223, no. 1, pp. 124-132, 2009.

[15] X. Han, "A class of general quartic spline curves with shape parameters," Computer Aided Geometric Design, vol. 28, no. 3, pp. 151-163, 2011. 